Article

\title{
Liquid Crystal Gel Reduces Age Spots by Promoting Skin Turnover
}

\section{Mina Musashi ${ }^{1, *}$, Ariella Coler-Reilly ${ }^{2}$, Teruaki Nagasawa ${ }^{1}$, Yoshiki Kubota ${ }^{1}$, Satomi Kato ${ }^{2}$ and Yoko Yamaguchi ${ }^{1,2}$}

1 NANOEGG ${ }^{\circledR}$ Research Laboratories, Inc., 2-16-1 Sugao, Miyamae-ku, Kawasaki, Kanagawa 216-8512, Japan; E-Mails: teruaki-nagasawa@nanoegg.co.jp (T.N.); yoshiki-kubota@nanoegg.co.jp (Y.K.); yoko-yamaguchi@nanoegg.co.jp (Y.Y.)

2 Institute of Medical Science, School of Medicine, St. Marianna University, 2-16-1 Sugao, Miyamae-ku, Kawasaki, Kanagawa 216-8512, Japan;

E-Mails: ariella@marianna-u.ac.jp (A.C.-R.); s2kato@marianna-u.ac.jp (S.K.)

* Author to whom correspondence should be addressed; E-Mail: mina-musashi@nanoegg.co.jp; Tel.: +81-44-978-4061; Fax: +81-44-978-5232.

Received: 7 March 2014; in revised form: 4 July 2014 / Accepted: 18 July 2014 /

Published: 25 July 2014

\begin{abstract}
Studies have shown that liquid crystals structurally resembling the intercellular lipids in the stratum corneum can beneficially affect the skin when applied topically by stimulating the skin's natural regenerative functions and accelerating epidermal turnover. In the present study, the effects of applying low concentrations of a liquid crystal gel of our own creation were evaluated using epidermal thickening in mouse skin as an assay for effective stimulation of epidermal turnover. A liquid crystal gel was also applied topically to human facial skin, and analysis was conducted using before-and-after photographs of age spots, measurements of $L^{*}$ values that reflect degree of skin pigmentation, single-layer samples of the stratum corneum obtained via tape-stripping, and measurements of trans-epidermal water loss that reflect the status of the skin's barrier function. The results suggested that cost-effective creams containing as low as $5 \%$ liquid crystal gel might be effective and safely sold as skin care products targeting age spots and other problems relating to uneven skin pigmentation.
\end{abstract}

Keywords: liquid crystal; skin turnover; intercellular lipids; stratum corneum; age spots; skin care 


\section{Introduction}

In the stratum corneum, the uppermost layer of the epidermis, intercellular lipids provide the semi-permeable barrier that is essential to the function of the skin [1]. These lipids are believed to be arranged in stacked membrane sheets and can be said to have a lamellar liquid crystal structure (Figure 1, top).

Figure 1. Diagram of lyotropic liquid crystal phase transitions illustrating that a lamellar phase liquid crystal can transition to either an unorganized phase or a bicontinuous cubic phase and back again.

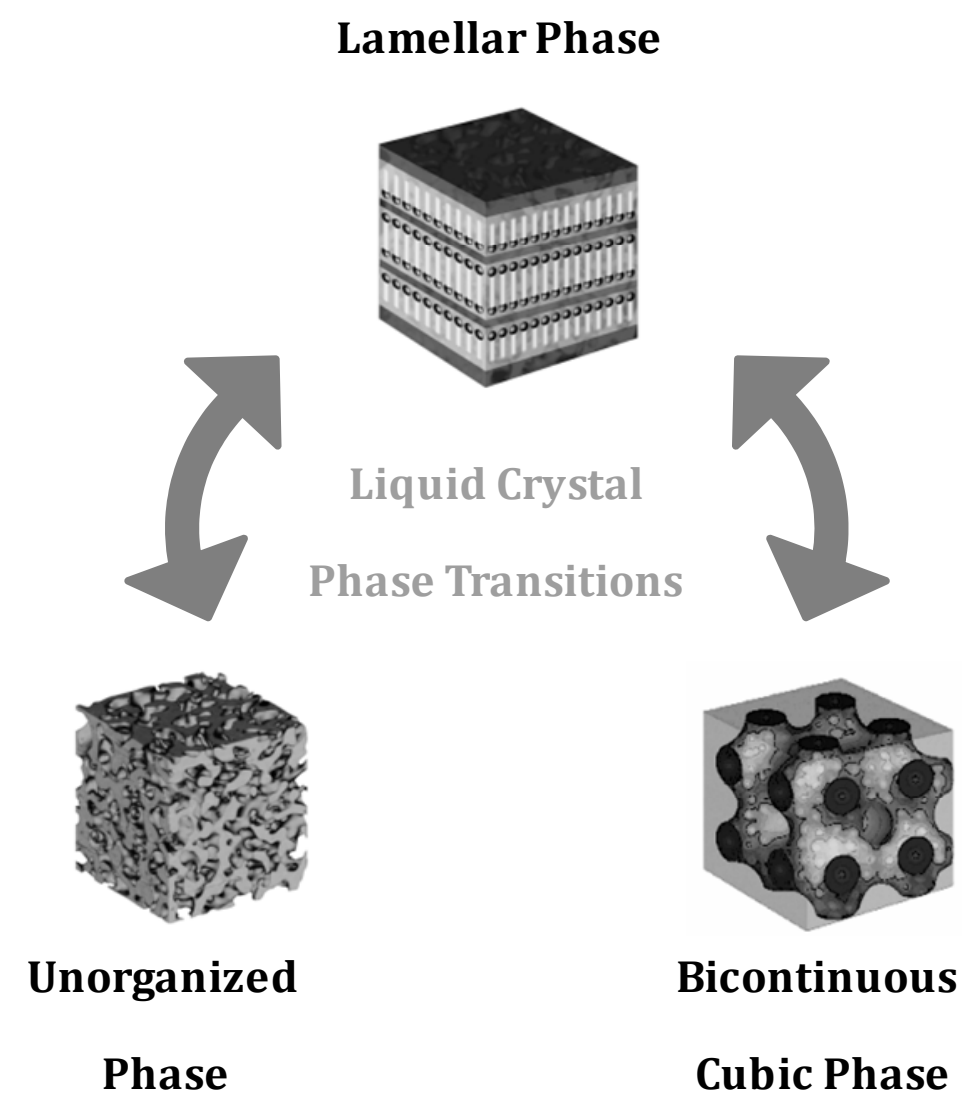

Previously, we have prepared lyotropic liquid crystal formulations using oil, water, and surfactant, and we have investigated the effects of topically applying such a substance as a liquid crystal gel (LCG) on skin [2]. Upon topical application to mammalian skin, we observed epidermal thickening, lightening of the skin, and a slight, transient increase in trans-epidermal water loss (TEWL). We inferred that the LCG stimulated epidermal cell turnover by temporarily disturbing the barrier function of the skin.

As illustrated in Figure 1, liquid crystals can transiently change form from lamellar to unorganized or bicontinuous cubic and then revert back again [3]. We inferred that lipid and/or aqueous components of the LCG may diffuse into the intercellular spaces of the epidermis and produce such a temporary change in the intercellular lipid structure, thereby disturbing the barrier function of the skin, generating the observed small spike in TEWL. We postulated that this disturbance in barrier function could signal to the body that the skin has been damaged and stimulate the skin's self-regeneration mechanisms, thereby accelerating epidermal turnover $[2,4]$. 
Rather than directly attacking problem areas in the skin such as age spots, the LCG exerts its influence indirectly by stimulating the skin's own natural self-repair and regeneration functions without damaging the skin in a visible or painful way [2]. This has obvious potential applications in skin care products, and in this study we targeted our research towards achieving such applications. We first sought to determine whether the LCG could be effective at concentrations low enough to be practical using the thickening of mouse epidermis as an assay for accelerated epidermal turnover, as described previously $[2,5,6]$. Following that, we directly quantified the ability of the LCG to erase age spots from human facial skin by analyzing before and after photographs and quantifiably measuring changes in skin pigmentation. We used photomicrographs of single-layer human stratum corneum samples to check for abnormalities in LCG-treated skin on a cellular level. We finished by conducting an experiment to confirm that LCG was not causing any long-term disruptions in skin barrier function. In all experiments, the skin was carefully observed for potential side effects such as irritation.

\section{Experimental Section}

\subsection{Preparation of Creams}

All creams were made by combining fixed amounts of hydrogenated lecithin and carbomer (980) from Nikko Chemicals Co., Ltd. (Tokyo, Japan), cholesterol from Nippon Fine Chemical Co., Ltd. (Osaka, Japan), xanthan gum from Dainippon Sumitomo Pharma Co., Ltd. (Osaka, Japan), dipropylene glycol from Showa Denko K.K. (Tokyo, Japan), polyethylene glycol (PEG-4000) from Sanyo Chemical Industries, Ltd. (Kyoto, Japan), cyclopentasiloxane from Shin-Etsu Chemical Co., Ltd. (Tokyo, Japan), potassium hydroxide from Wako Pure Chemical Industries (Osaka, Japan), and varying amounts water and LCG. The LCG was prepared as previously by combining Emulgen 2020HA (27.1 wt\%) from Kao Corporation (Tokyo, Japan), glycerol (36.8 wt\%) and squalane (19.6 wt\%) from Wake Pure Chemical Industries Ltd. (Tokyo, Japan), and water (16.5 wt\%) [2].

\subsection{Animal Studies}

\subsubsection{Animals}

All animals used in this study were male mice purchased at 5 weeks old from Japan SLC, Inc. (Shizuoka, Japan) and allowed to rest one week before applying treatments. Mice used for the experiment comparing various concentrations of LCG were ddY, while the mice used for measuring the change in epidermal thickness after applying 5\% LCG were HR-1. Cages were kept at 50\%-60\% humidity and $23 \pm 1{ }^{\circ} \mathrm{C}$ with light from 630 to $1830 \mathrm{~h}$ (12 h light-dark cycle), and animals were allowed ad libitum access to food and water. All experiments were carried out in accordance with the Guidelines for Animal Experimentation of St. Marianna University School of Medicine and approved by the Committee for Animal Experimentation. 


\subsubsection{Skin Treatments}

For the two ddY mice, in order to create a $2 \mathrm{~cm} \times 2 \mathrm{~cm}$ hairless area on each mouse on which to apply treatments, an area on the back slightly larger than $2 \mathrm{~cm} \times 2 \mathrm{~cm}$ was shaved with an electric razor three days before treatment. Following this, $30 \mathrm{mg}$ of one of the following three creams was applied to each hairless area twice per day for four days with two animals per treatment group: $0 \%$, $5 \%$, or $10 \%$ LCG cream. An additional two animals were shaved but left untreated.

For the three HR-1 mice, which were already hairless, $50 \mathrm{mg}$ of 5\% LCG cream was applied to the skin on the dorsal side once per day for four weeks. An additional three HR-1 mice were left untreated.

\subsubsection{Tissue Collection and Staining}

After the treatment, a single $1 \mathrm{~cm} \times 1.5 \mathrm{~cm}$ tissue section was cut from the treated area of each mouse, fixed in formalin, and embedded in paraffin. The tissue samples were then sliced into $4 \mu \mathrm{m}$ sections, mounted on slides, and treated with hematoxylin and eosin (H\&E) stain. Photomicrographs were then taken under a standard light microscope.

\subsection{Human Studies}

\subsubsection{Subjects}

A total of five middle-aged subjects of Japanese descent were enrolled in the human studies. All subjects willingly participated in the studies and were treated only after giving informed consent. Three subjects with facial age spots (39-year-old male, 40-year-old female, and 51-year-old female) consented to having their skin pigmentation analyzed before and after treatment using before-and-after photographs and $L^{*}$ values. Five subjects, those three and an additional two subjects (36-year-old male and 36-year-old female), donated stratum corneum samples and allowed their TEWL levels to be measured. All experiments were approved by the Ethical Committee for Cosmetics Tests at NANOEGG ${ }^{\circledR}$ Research Laboratories.

\subsubsection{Skin Treatments}

The 5\% LCG cream was applied to one side of each subject's face, while the other side was left untreated as the control. The cream was applied twice per day for a month. No other skin products affecting epidermal turnover or skin pigmentation were used by the subjects during the testing period. Photographs were taken before starting treatment and after the month of treatment for comparison.

\subsubsection{Skin Pigmentation Measurements}

Skin pigmentation intensities at the locations of the facial age spots were measured using a CM-700d spectrophotometer (Konica Minolta, Osaka, Japan). The level of skin pigmentation were expressed as $L^{*}$ values, which range from 0 to 100 , where $L^{*}=0$ would indicate black, and $L^{*}=100$ would indicate white [7]. Measurements were taken at the same spot before starting treatment and after the month of treatment for comparison. The fold change in $L^{*}$ values from before to after treatment for all participating subjects were calculated and analyzed for statistical significance. 


\subsubsection{Trans-Epidermal Water Loss (TEWL)}

After the one month of treatment, TEWL was measured on both the treated side of the face and the untreated side as a control. Measurements were taken using the Tewameter ${ }^{\circledR}$ TM300 (Integral, Tokyo, Japan).

\subsubsection{Tape-Stripping and Staining}

After the month of treatment, a small sheet of stratum corneum cells was obtained from each side of the face via tape-stripping as described previously [8] using Cellotape ${ }^{\circledR}$ from purchased from Nichiban Co., Ltd. (Tokyo, Japan). The pieces of tape were then attached skin-side-down to slides coated in dried $\operatorname{Scotch}^{\circledR}$ adhesive purchased from Sumitomo 3M Ltd. (Tokyo, Japan). After allowing the adhesive to set, the slides were submerged in xylene until the tape had separated from the slide leaving the skin cells behind. The slides were retrieved and left to dry at room temperature. The slides were stained with a $1: 1$ ratio of $0.5 \%$ brilliant green and $1 \%$ gentian violet for one minute and then washed thoroughly with water. Photomicrographs were taken under a standard light microscope.

\subsection{Statistical Analysis}

The data was analyzed using the student's $t$ test or the paired $t$ test. $p$ values less than 0.05 were considered statistically significant. Calculations were performed using " $R$ " software, version 2.13.1 [9].

\section{Results and Discussion}

\subsection{Low Concentration Liquid Crystal Gel (LCG) Thickens Epidermis in Mice}

A preliminary study was conducted to ascertain whether or not low concentration LCG creams could be sufficiently effective at accelerating epidermal turnover. Stained tissue sections obtained from mouse skin treated with various concentrations of the LCG are shown in Figure 2. The degree of epidermal thickening was correlated with the LCG concentration, confirming that the LCG is indeed the active ingredient causing the thickening. This thickening effect was visible at concentrations as low as 5\% LCG, indicating that products containing as little as 5\% LCG could be effective at stimulating epidermal turnover.

Next we sought to further evaluate the effects of the 5\% LCG by quantifying the epidermal thickening that occurs when the cream is applied daily for one month. As shown in Figure 3, we were able to demonstrate that application of 5\% LCG to mouse skin for four weeks produces a statistically significant increase in epidermal thickness $(n=3, p<0.01)$. These results are consistent with those of our previous study [2]; however, in this study we were able to quantify the increase in epidermal thickness and demonstrate its statistical significance for the first time. 
Figure 2. Liquid crystal gel (LCG) concentration-dependent increase in epidermal thickness is visible even at concentrations as low as 5\% and 10\% LCG as shown in these images of hematoxylin and eosin (H\&E)-stained mouse skin.

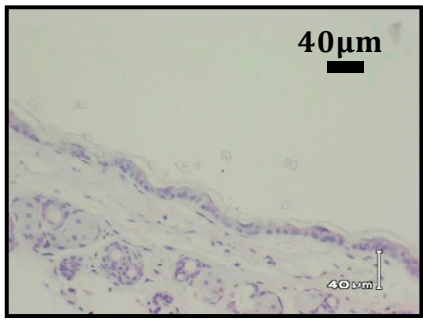

Untreated

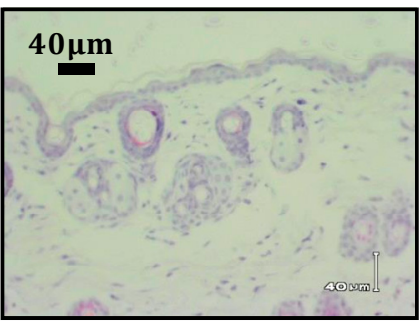

0\% LCG

Emulsion

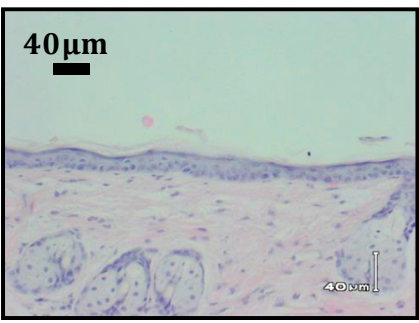

$5 \%$ LCG

Emulsion

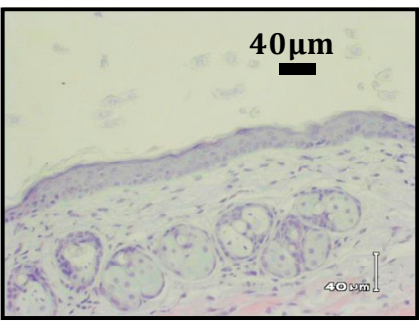

$10 \%$ LCG Emulsion

Figure 3. Five percent liquid crystal gel (LCG) induces statistically significant epidermal thickening when applied to mouse skin daily for four weeks. (a) Images of untreated and treated H\&E-stained skin; (b) Epidermal thickness of untreated versus treated skin $(n=3, * p<0.01)$.

(a)

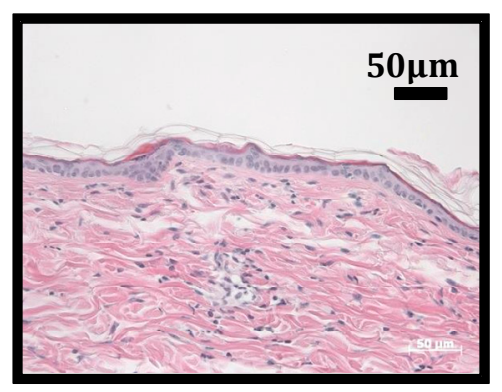

Untreated

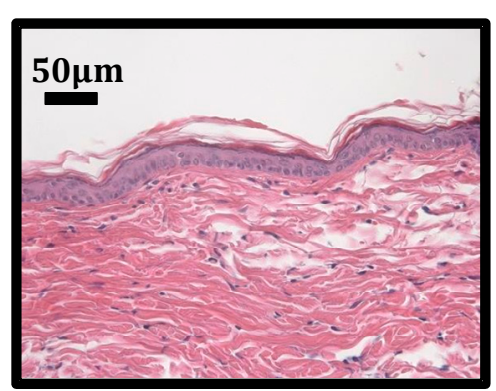

$5 \%$ LCG (b)

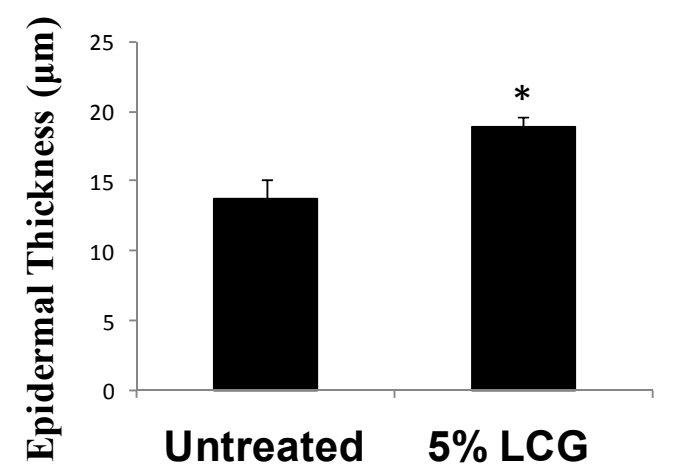

There are clear advantages to using a lower concentration LCG cream given that the cream remains effective at this low concentration. The creams tested in previous experiments were $10 \%, 20 \%$, and 30\% LCG creams, and it was noted that a high concentration of LCG can produce a mildly sticky, uncomfortable cream. Therefore, a lower concentration of LCG may be preferable. Additionally, a lower minimum effective concentration yields more cost-effective production.

\subsection{LCG Removes Spots on Human Skin}

Skin treated with the 5\% LCG cream became substantially clearer, as evidenced by analysis of before and after photographs (Figure $4 \mathrm{a}$ ) as well as the rise in $L^{*}$ values that occurred after one month of treatment ( $n=3, p<0.05$, Figure $4 \mathrm{~b}$ ). These results are consistent with the results of our previous study [2]; however, the previous study was limited to only one human subject with no tests of statistical significance, making the results of the present study much more reliable. As was the case with epidermal thickening, the disappearance of skin spots also indicates accelerated epidermal turnover. The photographs show no signs of adverse side-effects such as irritation, and all available evidence suggests that LCG can be employed in skin care products as a skin spot removal agent. 
Figure 4. Skin pigmentation is reduced upon applying 5\% liquid crystal gel (LCG) cream twice daily for one month. (a) Photographs of the same section of a subject's face before (left panel) and after (right panel) treatment, with the red circle denoting a particularly hyper-pigmented area of interest; (b) Reduction in pigmentation of age spots before and after treatment as measured by $L^{*}$ values denoting brightness $(n=3, p<0.05)$.

(a)

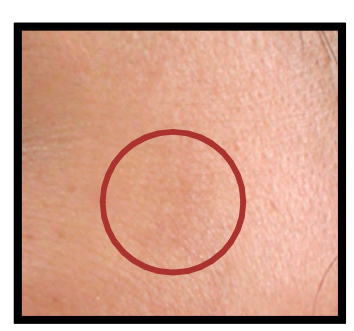

Before Treatment

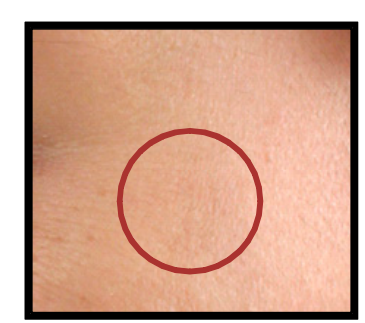

After Treatment (b)

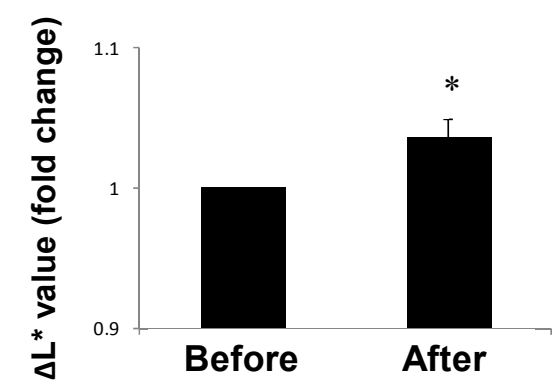

\subsection{LCG Does Not Over-Stimulate Skin Turnover or Reduce Skin Barrier Function}

There was some concern that the LCG may over-stimulate skin turnover, perhaps accelerating past the optimum level. Acceleration of epidermal turnover to a detrimental speed can produce keratinocytes that do not differentiate completely. In such cases, keratinocytes would not have time to lose their nuclei before ascending to the stratum corneum [10], and these nuclei would be visible in photomicrographs of tape-stripped samples of stratum corneum. As there were no nuclei visible in the photomicrographs of skin treated with 5\% LCG (Figure 5a), we have concluded that there is no evidence to suggest that 5\% LCG would over-stimulate skin turnover.

Another concern was that the LCG might disrupt the barrier function of the skin, which would be reflected as a rise in TEWL. Our measurements to date indicate that 5\% LCG has no long-term effect on TEWL ( $n=5, p=$ not significant, Figure $5 \mathrm{~b}$ ). Thus, there is currently no evidence to suggest that LCG has any deleterious effects on the skin.

Figure 5. No adverse effects were noted after using 5\% liquid crystal gel (LCG). (a) Representative images of tape-stripped sections of human stratum corneum stained with gentian violet-brilliant green after one month of treatment with (right panel) $5 \%$ LCG cream versus (left panel) untreated skin $(n=5)$; (b) Comparison of trans-epidermal water loss (TEWL) in skin treated with 5\% LCG as compared to untreated skin $(n=5$, $p=$ not significant).

(a)

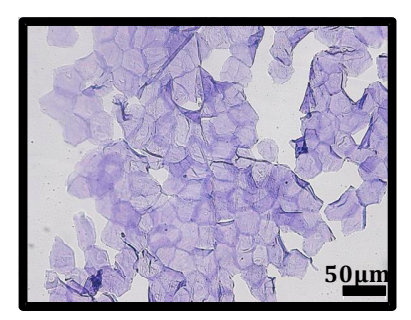

Untreated

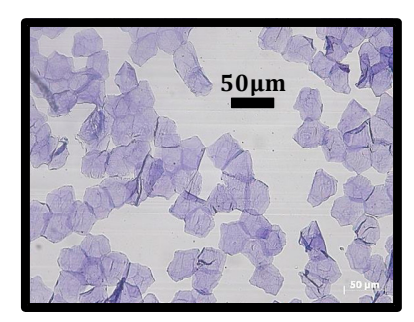

$5 \%$ LCG (b)

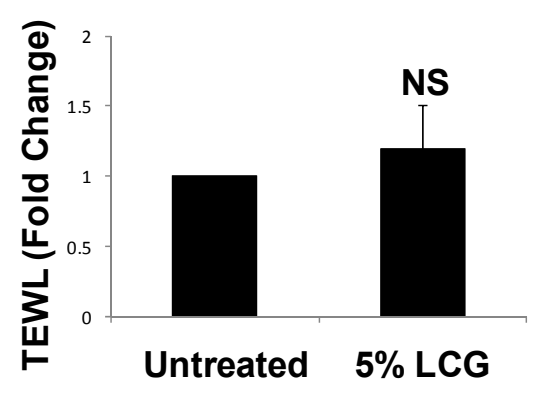




\section{Conclusions}

In conclusion, our results suggest that a relatively inexpensive, low concentration LCG can be safely used in skin products to even out skin pigmentation, visibly reducing the appearance of age spots. We postulate that the LCG works by transiently disrupting intercellular lipid structure, which stimulates the skin's natural regeneration functions and accelerates epidermal turnover. However, the precise mechanism underlying this phenomenon is not yet fully understood and remains to be investigated in future studies.

\section{Acknowledgments}

The authors thank Kimio Kawamura (NANOEGG ${ }^{\circledR}$ Research Laboratories, Inc., Kanagawa, Japan), Ryoko Nakagawa and Kazuyo Inoue (TBC Group Corporation, Ltd., Tokyo, Japan) for the technical support. This research was in part supported by funds provided by NANOEGG ${ }^{\circledR}$ Research $^{2}$ Laboratories, Inc. and the TBC Group Corporation, Ltd.

\section{Author Contributions}

M.M. and Y.Y. designed the study. M.M., T.N., Y.K., and S.K. performed the experiments. M.M., A.C.-R., and Y.Y. analyzed the data and wrote the paper.

\section{Conflicts of Interest}

Several authors of this publication including the lead author are employed at NANOEGG ${ }^{\circledR}$ Research Laboratories, Inc. and conduct research in areas of interest similar to the business interests of the company. This arrangement has been approved by the St. Marianna School of Medicine (Kanagawa, Japan).

\section{References}

1. Madison, K.C. Barrier function of the skin: "La raison d'être" of the epidermis. J. Invest. Dermatol. 2003, 121, 231-241.

2. Yamaguchi, Y.; Nagasawa, T. New nanotechnology for the guided tissue regeneration of skin-Potential of lyotropic liquid crystals. Pharmazie 2006, 61, 112-116.

3. Friberg, S.E. Micelles, microemulsions, liquid crystals, and the structure of stratum corneum lipids. J. Soc. Cosmet. Chem. 1990, 41, 155-171.

4. Lee, S.H.; Jeong, S.K.; Ahn, S.K. An update of the defensive barrier function of skin. Yonsei Med. J. 2006, 47, 293-306.

5. Iizuka, H. Epidermal architecture that depends on turnover time. J. Dermatol. Sci. 1995, 10, 220-223.

6. Yamaguchi, Y.; Nagasawa, T.; Nakamura, N.; Takenaga, M.; Mizoguchi, M.; Kawai, S.; Mizushima, Y.; Igarashi, R. Successful treatment of photo-damaged skin of nano-scale atRA particles using a novel transdermal delivery. J. Control. Release 2005, 104, 29-40.

7. Weatherall, I.L.; Coombs, B.D. Skin color measurements in terms of CIELAB color space values. J. Invest. Dermatol. 1992, 99, 468-473. 
8. Förster, M.; Bolzinger, M.A. Confocal Raman microspectroscopy for evaluating the stratum corneum removal by 3 standard methods. Skin Pharmacol. Physiol. 2011, 24, 103-112.

9. Ihaka, R.; Gentleman, R. R: A language for data analysis and graphics. J. Comput. Graph. Stat. 1996, 5, 299-314.

10. Willis, C.M.; Stephens, C.J. Epidermal damage induced by irritants in man: A light and electron microscopic study. J. Invest. Dermatol. 1989, 93, 695-699.

(C) 2014 by the authors; licensee MDPI, Basel, Switzerland. This article is an open access article distributed under the terms and conditions of the Creative Commons Attribution license (http://creativecommons.org/licenses/by/3.0/). 